\title{
Attempt to detect diamagnetic anisotropy of dust-sized crystal orientated to investigate the origin of interstellar dust alignment
}

\author{
T. Takeuchi, K. Hisayoshi, and C. Uyeda \\ Institute of Earth and Space Science, Graduate School of Science, Osaka University, Toyanaka, Osaka 560-0043, Japan
}

(Received December 1, 2011; Revised May 15, 2012; Accepted May 15, 2012; Online published March 12, 2013)

\begin{abstract}
Diamagnetic anisotropy $\Delta \chi_{\text {DIA }}$ was detected on a submillimeter-sized calcite crystal by observing the rotational oscillation of its magnetically stable axis with respect to the magnetic field direction. The crystal was released in an area of microgravity generated by a 1.5-m-long drop shaft. When the oscillations are observable, the present method can measure $\Delta \chi_{\text {DIA }}$ of crystal grains irrespective of how small they are without measuring the sample mass. In conventional $\Delta \chi$ measurements, the background signal from the sample holder and the difficulty in measuring the sample mass prevent measurement of $\Delta \chi_{\text {DIA }}$ for small samples. The present technique of observing $\Delta \chi_{\text {DIA }}$ of a submillimeter-sized single crystal is a step toward realizing $\Delta \chi_{\text {DIA }}$ measurements of micron-sized grains. The $\Delta \chi_{\text {DIA }}$ values of single micron-sized grains can be used to assess the validity of a dust alignment model based on magnetic torque that originates from the $\Delta \chi_{\text {DIA }}$ of individual dust particles.
\end{abstract}

Key words: Dust alignment, diamagnetic anisotropy, magnetic rotational oscillation.

\section{Introduction}

The spatial distributions of inter- and circumstellar magnetic fields are commonly estimated from the polarization of visible and infrared light from stars; this polarization is considered to result from the magnetic alignment of the dust particles that are present in various regions (e.g. Spizer, 1978). The origin of grain alignment in the diffuse interstellar region had been explained by a paramagnetic relaxation of the grains; here the grain possesses angular momentum due to its collisions with the gas particles (Davis and Greenstein, 1951). The model was improved by assuming a spin-up of the grain caused by the ejection of molecular hydrogen from the grain surface (the "pinwheel mechanism", Purcell, 1979), however quantitative inconsistencies remained between the improved model and observed interstellar conditions (e.g. Lazarian and Draine, 1999). A possibility of grain alignment based on a radiative torque was proposed (Dolginov and Mitrofanov, 1976); the torque was effective on irregularly-shaped grains with geometrical "helicity". The efficiency of the radiative torque was improved by assuming super-paramagnetic inclusions in the grains (Lazarian and Hoang, 2008). The magnetic properties were studied for metallic inclusions formed in various types of silicate matrix (Djouadi et al., 2007); their microstructures resembles those of glass with embedded metal and sulphides found in the interplanetary dust particles. Large remnant magnetization that derives from magnetic inclusions contained in natural olivine crystals are reported (Belley et al., 2009); olivine is recognized as one of the major minerals contained in meteorites and circumstel-

Copyright (c) The Society of Geomagnetism and Earth, Planetary and Space Sciences (SGEPSS); The Seismological Society of Japan; The Volcanological Society of Japan; The Geodetic Society of Japan; The Japanese Society for Planetary Sciences; TERRAPUB.

doi:10.5047/eps.2012.05.007 lar dusts. It was pointed out that the large magnetizations of these materials are effective in realizing grain alignment in terms of the above-mentioned mechanisms (Djouadi et al., 2007). In high-density regions, the mechanism of dust alignment is still unclear, because the effects of the abovementioned mechanism are reduced when dust is in thermal equilibrium with the gas medium (Whittet, 1992).

A simple mechanism has been proposed that is based on the balance between the rotational Brownian energy and the magnetic anisotropy energy of a dust particle. Unlike the conventional models, this mechanism is effective even when gas and dust are in a thermal equilibrium state. The magnetic anisotropy may be caused by paramagnetic anisotropy $\Delta \chi_{\text {PARA }}$ and/or by diamagnetic anisotropy $\Delta \chi_{\text {DIA }}$ (Uyeda et al., 1991, 1993). The amount of $\Delta \chi_{\text {PARA }}$ required for dust alignment has been quantitatively discussed based on laboratory experiments using orthopyroxene grains containing a low concentration $(\sim 1 \mathrm{~mol} . \%)$ of $\mathrm{Fe}^{3+}$ ions; orthopyroxene has been detected in circumstellar regions by infrared emission spectroscopy (Honda et al., 2003). Since the temperature dependence of $\Delta \chi_{\text {PARA }}$ follows the Curie law, the field intensity required to cause alignment is expected to be relatively low at the low temperatures that are assumed to exist in the outer regions of protoplanetary disks (Uyeda et al., 2003a, 2005). Correlation between interstellar polarization and dust temperature was confirmed for 14 lines of sight towards the Pleiades cluster (Matsumura et al., 2011), which was compatible with an alignment model based on the radiative torques. The observed correlation between temperature and polarization is effective to examine the efficiency of the above-mentioned models based on magnetic torques, since large temperature dependences are expected for the magnetizations of dust materials (Uyeda et al., 2003a; Belley et al., 2009).

In diffuse clouds, the concentrations of magnetic ions in 

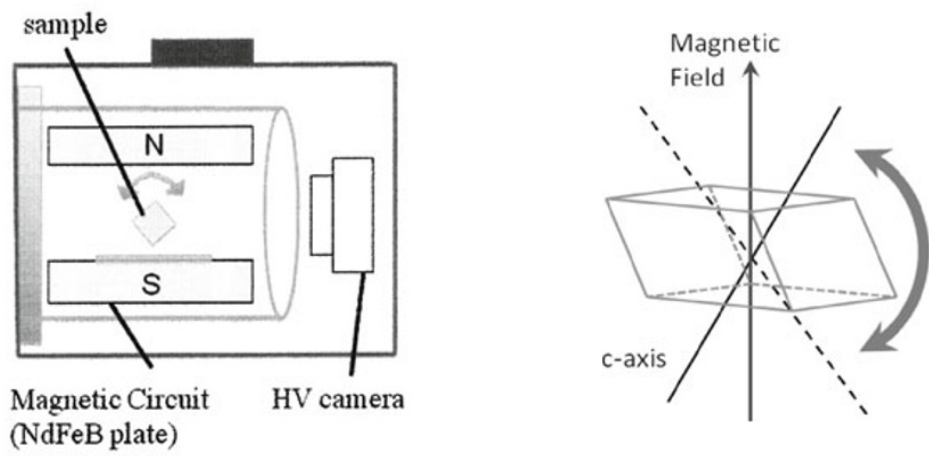

Fig. 1. Schematic view of the experimental setup to observe field-induced rotational oscillation using a chamber-type drop shaft. The diagram on the right shows the geometric relationship between field direction and axis of rotation; the crystalline $c$-axis is magnetically unstable.

the dust particles are considered to be too low to cause dust alignment. In these regions, dust alignment may be caused by $\Delta \chi_{\text {DIA }}$ (Chihara et al., 1988; Uyeda et al., 2003b). According to a recent model based on experimental $\Delta \chi_{\text {DIA }}$ data, materials generally possess an intrinsic $\Delta \chi_{\text {DIA }}$. Recently, there have been various attempts to determine the effective susceptibility of a small diamagnetic particle. Significant increases in $\Delta \chi_{\text {DIA }}$ with decreasing crystal size have been reported recently for various materials. To assess the validity of the above-mentioned alignment model, it is essential to obtain the effective $\Delta \chi_{\text {DIA }}$ of actual dust particles. However, it is difficult to determine $\Delta \chi_{\text {DIA }}$ using conventional methods for samples with submillimeter diameters. In the present study, $\Delta \chi_{\text {DIA }}$ of submillimeter calcite crystals was obtained for the first time by measuring the period of rotational oscillations caused by the fieldinduced anisotropy energy. The crystals were released in a microgravity $(\mu \mathrm{G})$ area generated by a short drop shaft. The size dependence of $\Delta \chi_{\text {DIA }}$ is then investigated using calcite crystals of different sizes. The possibility of extending these measurements to micron or submicron sizes is discussed based on the obtained results.

\section{Experimental}

In the present study, $\mu \mathrm{G}$ conditions were produced using a 1.5-m-long drop shaft, which was designed and constructed at the Graduate School of Science, Osaka University. The drop shaft produced $\mu \mathrm{G}$ for a duration of $0.5 \mathrm{~s}$. An experimental setup developed to measure magnetic rotation was installed in the drop shaft. It was in the form of a rectangular box that had internal dimensions of $35 \mathrm{~cm} \times 30 \mathrm{~cm} \times 20 \mathrm{~cm}$. Routine $\mu \mathrm{G}$ experiments using the above-mentioned short drop shaft were difficult for larger box sizes. Prior to the experiment, submillimeter calcite crystals were inserted in the center of a homogeneous field generated by a magnetic circuit consisting of two $\mathrm{NdFeB}$ magnetic plates with dimensions of $2.5 \mathrm{~cm} \times 2.0 \mathrm{~cm} \times 0.6 \mathrm{~cm}$. The magnetic field intensity at the center of the circuit was $0.63 \mathrm{~T}$. The sample was supported by a sample stage. The calcite crystals were cut from a high-quality natural single crystal (Ecke, Iceland). Magnetic and chemical analyses performed in a previous study (Uyeda et al., 1993) revealed that it had a paramag- netic ion concentration of less than $1 \mathrm{ppm}$. The published $\Delta \chi_{\text {DIA }}$ value of calcite is $4.05 \times 10^{-8} \mathrm{emu} / \mathrm{g}$ and the crystal $c$-axis is a magnetically unstable axis (Uyeda et al., 1993). The experimental setup was enclosed in a vacuum chamber that had Pyrex walls to allow the sample motion to be observed from outside by a high-vision video camera (Panasonic, HDC-SD3-S) that had time and spatial resolutions of $0.033 \mathrm{~s}$ and $0.004 \mathrm{~cm}$, respectively.

The drop box was attached to the laboratory ceiling. About $0.05 \mathrm{~s}$ after the beginning of $\mu \mathrm{G}$, the sample was released from the sample stage in the homogeneous field region with a negligibly small angular momentum. The low pressure of the medium in the chamber $(P \approx 100$ $\mathrm{Pa}$ ) resulted in a low viscous drag. Prior to performing the $\mu \mathrm{G}$ experiments using the chamber drop shaft, operational tests of the above-mentioned setup were conducted to observe oscillation in a drop shaft at the National Institute of Advanced Technology AIST, which had a $\mu \mathrm{G}$ duration of $1.35 \mathrm{~s}$ and a residual gravitational acceleration of below 50 Gal. These tests confirmed that images of rotational oscillation could be recorded within a time 0.5 s (i.e., the $\mu \mathrm{G}$ duration of the chamber drop shaft).

\section{Results and Discussion}

In the present study, a diamagnetic single crystal is released in a low-pressure $\mu \mathrm{G}$ area with a homogeneous magnetic field $\boldsymbol{B}$ and the initial angular momentum of the sample is negligibly small. Under these conditions, the magnetically stable axis causes rotational motion with respect to $\boldsymbol{B}$. The rotation obeys

$$
I\left(d^{2} \theta / d t^{2}\right)=-m \Delta \chi_{\mathrm{DIA}} B^{2} \sin 2 \theta,
$$

where $\theta$ denotes the angle of the stable axis with respect to $\boldsymbol{B} ; I$ is the moment of inertia of the crystal. When the initial value of $\theta$ is sufficiently small, the period of rotational harmonic oscillation of the crystal can be written as:

$$
\tau \approx 2 \pi\left(I^{-1} \Delta \chi_{\text {DIA }}\right)^{1 / 2} B^{-1} .
$$

When the values of $B$ and $I m^{-1}$ are known, the above equation can be used to determine the intrinsic $\Delta \chi_{\text {DIA }}$ of the material, only by measuring $\tau$. The sensitivity of measuring a small $\Delta \chi_{\text {DIA }}$ can be improved by increasing $B$ and/or by 
Table 1. Specifications of calcite crystals measured in the present study.

\begin{tabular}{cccc}
\hline Sample No. & Mass $(\mathrm{mg})$ & Period of oscillation $\tau(\mathrm{ms})$ & $\Delta \chi_{\text {DIA }}\left(\times 10^{-8} \mathrm{emu} / \mathrm{g}\right)$ \\
\hline 1 & $0.090 \pm 0.009$ & $50 \pm 2$ & $5.5 \pm 0.9$ \\
2 & $1.162 \pm 0.167$ & $121 \pm 5$ & $5.1 \pm 1.0$ \\
3 & $1.442 \pm 0.176$ & $169 \pm 4$ & $2.8 \pm 0.4$ \\
4 & $4.325 \pm 0.377$ & $232 \pm 7$ & $2.9 \pm 0.3$ \\
5 & $5.208 \pm 0.368$ & $215 \pm 13$ & $3.5 \pm 0.6$ \\
\hline
\end{tabular}

increasing $\tau$. Using the above equation, the $\tau$ values of various millimeter-sized samples were measured to obtain their $\Delta \chi_{\text {DIA }}$ (Uyeda et al., 1993, 2005).

In the present study, calcite crystals underwent rotational oscillation with the geometry shown on the right-hand side of Fig. 1. In these oscillations, the equilibrium direction of the crystal $c$-axis was perpendicular to the field, since the $c$-axis was the magnetically unstable axis. The values of $\tau$ and $\mathrm{Im}^{-1}$ of the crystal were estimated from the images obtained using the high-vision camera. As mentioned before, the value of $B$ was $0.63 \mathrm{~T}$ in the homogeneous area of the circuit. By substituting these three values into Eq. (2), the $\Delta \chi_{\text {DIA }}$ of a single crystal grain can be obtained. Rotational oscillations of five calcite crystal grains with different masses $m$ were measured. Table 1 lists the obtained values of $\tau, \Delta \chi_{\text {DIA }}$ and $m$. Whereas, Fig. 2 shows a plot of $\Delta \chi_{\text {DIA }}$ against $m$. No significant dependence of $\Delta \chi_{\text {DIA }}$ on $m$ is observed for calcite above the experimental error. This mass independence is expected from Eq. (2). To quantitatively examine the slight enhancement of $\Delta \chi_{\text {DIA }}$ on reducing $m$, it is essential to improve the accuracy of $\mathrm{Im}^{-1}$ values to reduce the experimental errors in Fig. 2. Submillimeter samples are not expected to show a significant increase in $\Delta \chi_{\text {DIA }}$ with decreasing size.

It has been considered that circumstellar magnetic fields play a major role in the evolution of protoplanetary disks. To quantitatively examine the validity of various models on disk evolution based on the magnetic field, it is necessary to accurately measure the polarimetric distribution with a high spatial resolution. Near-infrared imaging polarimetry has been performed for massive young objects (Momose et al., 2001). In addition, young stellar objects have been imaged by near-infrared (Lucas et al., 2004) and submillimeter (Tamura et al., 1999) imaging of polarization. It is expected that a polarimetry survey with a high spatial resolution is realized by an Atacama Large Millimeter Array project. As mentioned above, it is essential to determine the dust alignment mechanism to obtain reliable field directions from polarimetry data, because the dust alignment mechanism determines the relationship between the magnetic field direction and the polarization. As mentioned before, the alignment mechanism based on relaxation of the paramagnetic moment of dust is not quantitatively approved, as yet, in the planetary formation regions where dust and gas are in thermal equilibrium (Whittet, 1992). The high angular momentum relative to that of the thermal equilibrium state assumed in the mechanism is realized only in diffuse interstellar regions. Hence, an alternative model was proposed that describes the origin of magnetic alignment based on the anisotropy of magnetic susceptibility (Uyeda et al., 2003b, 2010).

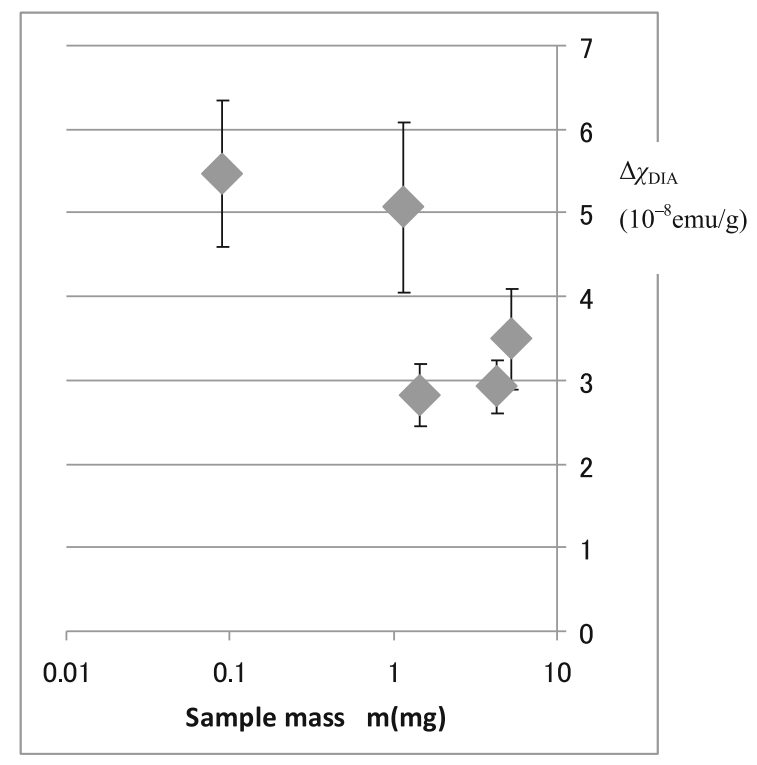

Fig. 2. Relationship between $\Delta \chi_{\text {DIA }}$ and mass $m$ measured for 5 calcite crystals. The $\Delta \chi_{\text {DIA }}$ and $m$ are also given in Table 1 . The errors are mainly due to the moment of inertia of the crystals and the uncertainty of observed $\tau$ values (see Table 1).

Magnetic alignment of diamagnetic particles has received much attention in connection with the processing of functional materials (e.g. Maret and Dransfield, 1985); an aggregate of grains with their crystal axes oriented in a single direction is expected to realize additional functionality along the oriented direction. The alignment process has been qualitatively analyzed by assuming a balance between the anisotropy energy, $1 / 2 m \Delta \chi B^{2}$, and the rotational Brownian motion, $1 / 2 k_{B} T$; here, $\Delta \chi$ denotes magnetic anisotropy ( $\Delta \chi_{\text {PARA }}$ and/or $\left.\Delta \chi_{\text {DIA }}\right)$. In order to estimate the field intensity required to achieve alignment, a new parameter $\left(B_{S}\right)$ was proposed to describe an ensemble of diamagnetic particles dispersed in a liquid medium at temperature $T$ (Yamagishi et al., 1989). $B_{s}$ is defined as the field intensity at which the order parameter $\langle M\rangle \approx 0.78$, where $\langle M\rangle$ is the average value of the function $1 / 2\left(3 \cos ^{2} \theta-1\right)$ for a grain ensemble. In the above function, $\theta$ is the angle between the magnetically stable axis of a grain and $\boldsymbol{B}$. The value of $B_{s}$ was directly calculated for a Langevin process (Langevin and Curie, 1910) to be

$$
B_{s}=\left(15 k_{B} T / m \Delta \chi\right)^{1 / 2} \text {. }
$$

This equation indicates that $B_{s}$ decreases with decreasing $T$ and with increasing $m$ or $\Delta \chi$. The theoretical value of $B_{s}$ is typically calculated by substituting experimentally measured $m, \Delta \chi$, and $T$ values into Eq. (3), while an experi- 
mental estimate of $B_{s}$ is independently obtained from the measured $\langle M\rangle-B$ relationship. When these two values of $B_{s}$ agree with each other, the grain alignment is considered to be driven by a Langevin process. The abovementioned agreement was examined for various minerals contained in meteorites and circumstellar dust (Uyeda et al., 2005a, 2010), and the possibility of grain alignment caused by $\Delta \chi_{\text {PARA }}$ was discussed on the basis of the agreement.

The dust alignment caused by $\Delta \chi_{\text {DIA }}$ is considered to be less realistic compared with an alignment caused by $\Delta \chi_{\text {PARA }}$, since the calculated $B_{S}$ value based on $\Delta \chi_{\text {DIA }}$, obtained by bulk crystal measurement, is considerably large compared to the astronomical magnetic field. As mentioned before, significant increases of $\Delta \chi_{\text {DIA }}$ with decreasing crystal size was reported recently for some of the ceramic materials. If the diamagnetic anisotropy energy due to the enhanced $\Delta \chi_{\text {DIA }}$ of the micron-sized material becomes comparable to the Brownian motion energy at an astronomical condition, partial alignment of dusts may occur by $\Delta \chi_{\text {DIA }}$. Unlike many of the conventional models that are based on super-thermal effects, the mechanism based on diamagnetic anisotropy energy is effective in the dense region where the evolution of proto-planetary discs take place. Furthermore, the mechanism is free of various specific magneticstructures assumed in the previous models; the magneticstructures were composed of small magnetic inclusions.

The practicability of considering grain alignment by diamagnetic anisotropy energy can be examined, by evaluating the quantitative amount of $\Delta \chi_{\text {DIA }}$ enhancement at the micron-size level. The new method for measuring $\Delta \chi_{\text {DIA }}$ in $\mu \mathrm{G}$ conditions, as described in Fig. 1, is free of a restoration torque since the sample is not suspended by a fiber. Furthermore, the method does not require measuring the sample mass. These two factors limit the minimum sample size of conventional methods for measuring $\Delta \chi_{\text {DIA }}$ under normal gravity. This implies that the present method, based on the observation of rotational oscillation, can measure the diamagnetic anisotropy of samples irrespective of how small they are. The minimum sample size could potentially be of the order of nanometers if an ultraviolet fluorescence microscope is introduced in the drop box. In the above setup, the sample rotation is observed by the ultraviolet emission from a fluorescent bead that is attached to the sample. The minimum diameter of this bead is presently several nanometers. Hence, $\Delta \chi_{\text {DIA }}$ is measurable for a particle that has a diameter greater than $10 \mathrm{~nm}$. As a step toward realizing measurements at $\mu \mathrm{m}$ and $\mathrm{nm}$ scales, it is important to improve the accuracy of $\mathrm{Im}^{-1}$ measurements of submillimeter samples (see Fig. 2). The demand for measuring $\Delta \chi_{\text {DIA }}$ of small single particles is increasing due to the growing interest in nano-sized materials in various research fields. Finally, the present experimental system to observe the free magnetic rotation of a single particle is applicable to examine the effectiveness of various types of magnetic torques that has been proposed in the conventional models to explain dust alignment. In order to perform these examinations, it is essential to realize the above-mentioned observation for micron and submicron sized particles.

\section{Conclusion}

1. When the period of field-induced rotational oscillation $\tau$ is observed for a crystal that is subject to $\mu \mathrm{G}$ conditions, its diamagnetic anisotropy $\Delta \chi_{\text {DIA }}$ (per unit mass) can be measured for any crystal, no matter how small they are. This is because the method does not require a sample holder and does not need to measure the sample mass.

2. By employing a compact drop shaft that produces $\mu \mathrm{G}$ conditions for $0.5 \mathrm{~s}, \Delta \chi_{\text {DIA }}$ was detectable for submillimeter calcite crystals by measuring $\tau$. No significant mass dependence was observed for the $\Delta \chi_{\text {DIA }}$ values at this size.

3. The validity of a dust alignment model based on diamagnetic anisotropy is quantitatively examined by obtaining $m \Delta \chi_{\text {DIA }}$ values at sizes equivalent to those of dust particles. This is because the field intensity to achieve alignment is uniquely determined by $m \Delta \chi_{\text {DIA }}$ of the dust and the medium temperature $T$. We are currently seeking to improve these $\Delta \chi_{\text {DIA }}$ measurements under $\mu \mathrm{G}$ conditions to enable $\Delta \chi_{\text {DIA }}$ to be measured for micron-sized crystals.

\section{References}

Belley, F., E. C. Ferré, F. Martın-Hernândez, M. J. Jackson, M. D. Dyar, and E. J. Catlos, The magnetic properties of natural and synthetic (Fex, $\mathrm{Mg} 1$ - x)2 $\mathrm{SiO}_{4}$ olivines, Earth Planet. Sci. Lett., 284, 516, 2009.

Chihara, H., C. Uyeda, A. Tsuchiyama, and T. Yamanaka, The magnetic ordering of graphite grains and an experimental application to grain alignment, Publ. Astron. Soc. Jpn., 50, 149-154, 1988.

Davis, L. Jr. and J. L. Greenstein, The polarization of starlight by aligned dust grains, Astrophys. J., 114, 206, 1951.

Djouadi, Z., J. Gattacceca, L. D’Hendecourt, P. Rochette, A. P. Jones, C. Davoisne, H. Leroux, and J. Borg, Ferromagnetic inclusions in silicate thin films: Insights into the magnetic properties of cosmic grains, Astron. Astrophys., 468, L9-L12, 2007.

Dolginov, A. Z. and I. G. Mitrofanov, Orientation of cosmic dust grains, Astrophys. Space Sci., 43, 291, 1976.

Honda, M., H. Kataza, Y. K. Okamoto, T. Miyata, T. Yamashita, S. Sako, S. Takubo, and T. Onaka, Detection of crystalline silicates around the T Tauri star Hen 3-600A, Astrophys. J., 585, L59-L63, 2003.

Langevin, P. and P. Curie, Magnetisme et theorie des electrons, CP Acad. Sci. Paris, 151, 331-368, 1910.

Lazarian, A. and B. T. Draine, Thermal flipping and thermal trapping: New elements in grain dynamics, Astrophys. J., 516, L37, 1999.

Lazarian, A. and T. Hoang, Alignment of dust with magnetic inclusions: Radiative torques and superparamagnetic barnett and nuclear relaxation, Astrophys. J., 676, L25, 2008.

Lucas, P. W., M. Fukagawa, M. Tamura, and A. Beckford, High-resolution imaging polarimetry of HL Tau and magnetic field structure, Mon. Not. R. Astron. Soc., 352, 1347-1364, 2004.

Maret, G. and K. Dransfield, Biomolecules and polymers in high steady magnetic field, Topics Appl. Phys., 57, 144-204, 1985.

Matsumura, M., Y. Kameura, K. S. Kawabata, H. Akitaya, M. Isogai, and M. Seki, Correlation between interstellar polarization and dust temperature: Alignment of grains by radiative torques is ubiquitous?, Publ. Astron. Soc. Jpn., 63, L43, 2011.

Momose, M., M. Tamura, O. Kameya, J. S. Greaves, A. Chrysostomou, J. H. Hough, and J. I. Morino, Submillimeter imaging polarimetry of the NGC 7538 region, Astrophys. J., 555, 855-862, 2001.

Purcell, E. M., Suprathermal rotation of interstellar grains, Astrophys. J., 231, 404, 1979.

Spizer, L. Jr., Physical Properties in the Interstellar Medium, Wiley \& Sons Inc., New York, 1978.

Tamura, M., J. H. Hough, J. S. Greaves, J. I. Morino, A. Chrysostomou, W. S. Holland, and M. Momose, First detection of submillimeter polarization from T Tauri stars, Astrophys. J., 525, 832-836, 1999.

Uyeda, C., T. Takeuchi, A. Yamagishi, and M. Date, Diamagnetic orientation of clay mineral grains, J. Phys. Soc. Jpn., 60, 3234-3237, 1991. 
Uyeda, C., T. Takeuchi, A. Yamagishi, T. Yamanaka, A. Tsuchiyama, and M. Date, Diamagnetic anisotropy of sheet silicates, Phys. Chem. Miner., 20, 369-374, 1993.

Uyeda, C., M. Sakakibara, and K. Tanaka, Temperature dependence observed of magnetic rotation process of micron sized kaolinite, Phys. Chem. Miner., 30, 425-429, 2003a.

Uyeda, C., T. Komatzu, K. Sakakibara, and K. Tanaka, Magneto-rotation experiments of micron-sized particles dispersed in a diffused gas medium oriented to reproduce the dust alignment in the interstellar region, Astron. Astrophys., 400, 805, 2003 b.

Uyeda, C., R. Takashima, and K. Hiraoka, Magnetic alignment of nonmagnetic mica crystals achieved at low field intensity due to high concentration of paramagnetic ions, Jpn. J. Appl. Phys., 44, L371-L374, 2005.

Uyeda, C., R. Takashima, and K. Tanaka, Magneto-rotation of nonmag- netic micro-crystals caused by diamagnetic anisotropy, Appl. Phys. Lett., 28, 094103, 2004.

Uyeda, C., K. Hisayoshi, and S. Kanou, Magnetic alignment of nonmagnetic silicates caused by paramagnetic anisotropy: Origin of polarization observed in planetary formation region, Earth Planets Space, 62, 99-103, 2010.

Whittet, D. C. B., Dust in the Galactic Environment, p. 110, Institute of Physics Publishing Bristol, 1992.

Yamagishi, A., T. Takeuchi, H. Higashi, and M. Date, Diamagnetic orientation of polymerized molecules under high magnetic-field, J. Phys. Soc. Jpn., 58, 2280-2283, 1989.

T. Takeuchi, K. Hisayoshi, and C. Uyeda (e-mail: uyeda@ess.sci.osakau.ac.jp) 